\title{
Learning the Exciton Properties of Azo-dyes
}

\author{
Sergi Vela, Alberto Fabrizio, Ksenia R. Briling and Clémence Corminboeuf*
}

Institute of Chemical Sciences and Engineering, École Polytechnique Fédérale de Lausanne (EPFL), Laboratory for Computational Molecular Design, CH-1015 Lausanne, Switzerland

\section{SUPPORTING INFORMATION}

\section{Table of Contents}

S1. Fragment Library........................................................................................................................................................

S2. Identification of the productive $\mathrm{n} \pi *$ and $\pi \pi^{*}$ states in ab initio computations...................................................

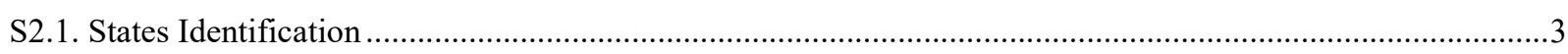

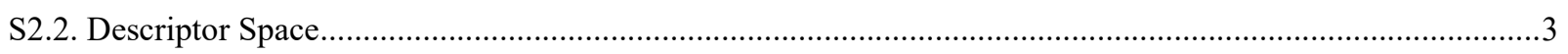

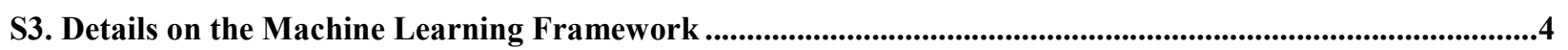

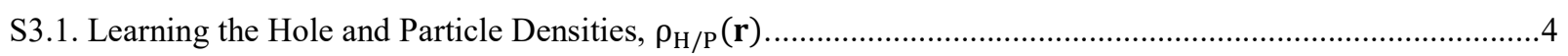

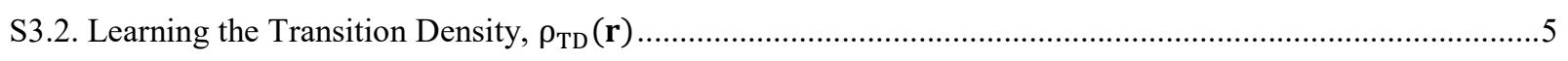

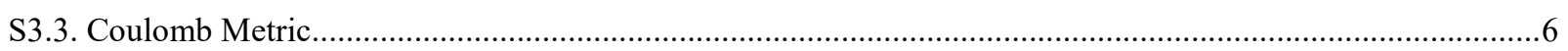

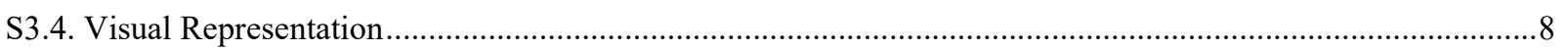

S3.5. Learning Curve of the Hole and Particle Distributions .............................................................................

S4. Comparison of Predicted vs. ab initio Descriptors ....................................................................................................10

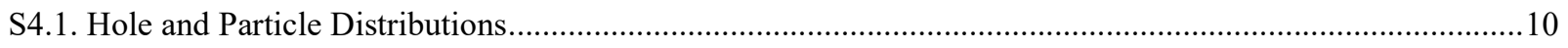

S4.2. Non-local nature of the $\pi \pi^{*}$ state, and impact on the predicted $\mathrm{H}_{\mathrm{R}_{1}}^{\pi{ }^{*}}$ values..........................................10

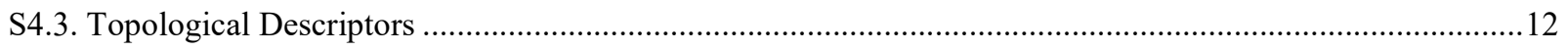

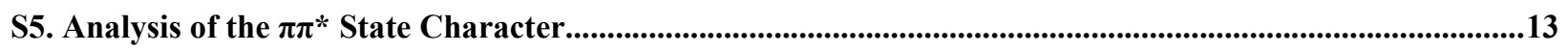

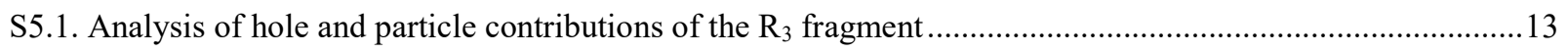

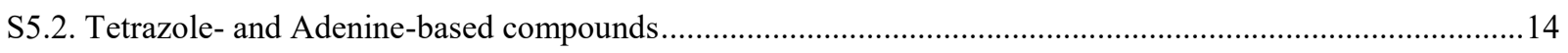

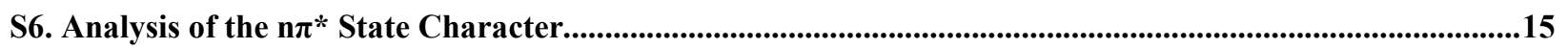

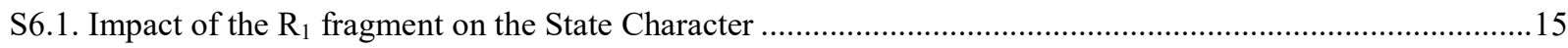

References ……...........................................................................................................................................................15 


\section{S1. Fragment Library}

We generated an initial database of 3432 azoheteroarenes from the combination of the azo moiety with four other fragments, labelled $R_{1-4}$ (see Table $S 1$ ). The first $\left(R_{1}\right)$ and second $\left(R_{2}\right)$ fragments are the adjacent rings, while the third $\left(R_{3}\right)$ and fourth $\left(R_{4}\right)$ are their peripheral substituents. These fragments have been selected to promote diversity in the $\pi \pi^{*}$ transition. For the sake of a balanced dataset and greater chemical diversity, we have included moieties with $\mathrm{S}$ and $\mathrm{O}$ atoms, five and six-membered rings substituted in ortho- and meta positions, and even fused rings.

Table S1. List and code of the fragments $\mathrm{R}_{1-4}$ included in the library. The fragments are combined following Scheme 1 to obtain the 3432 compounds studied in this work.

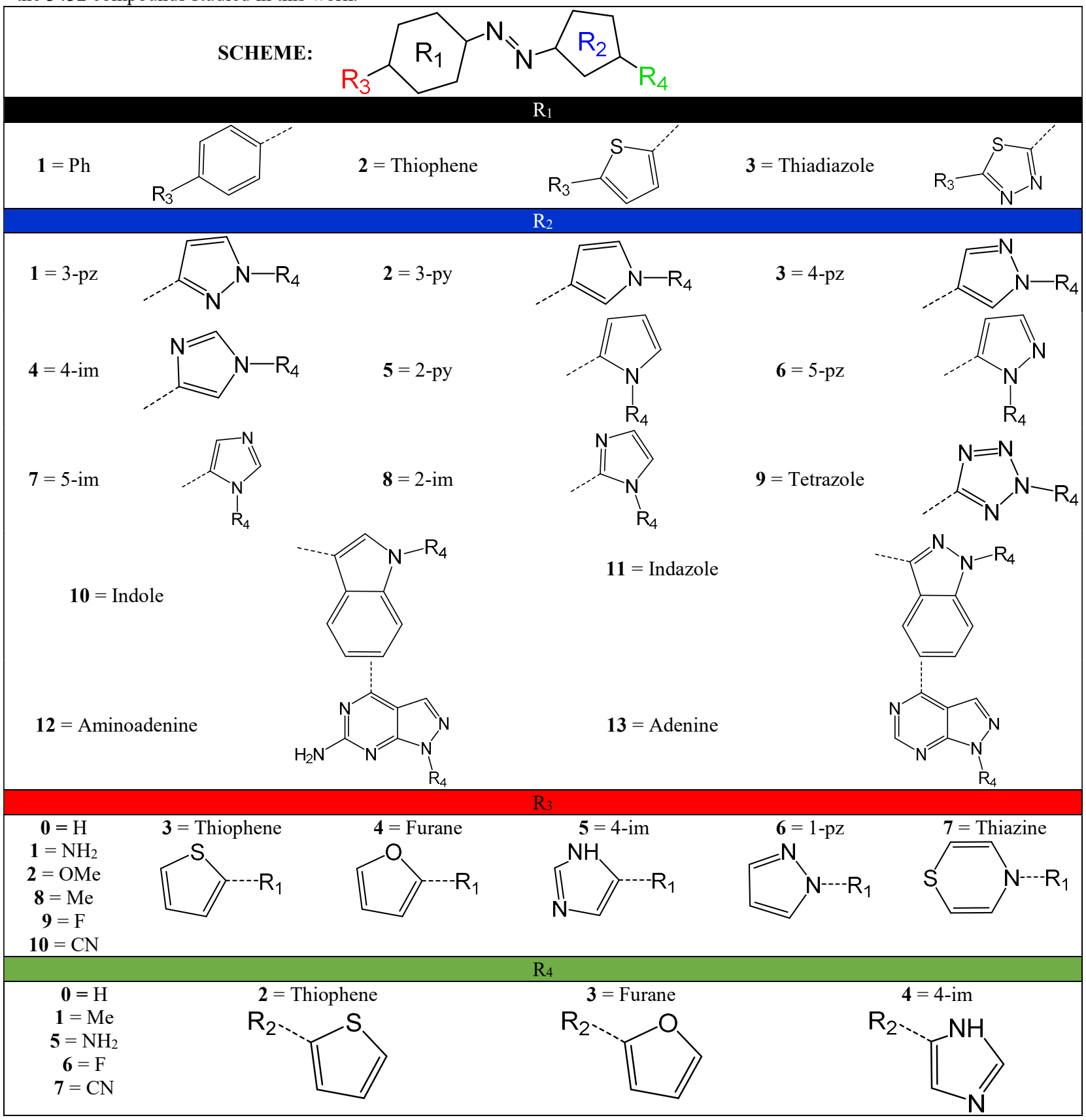




\section{S2. Identification of the productive $\mathrm{n} \pi *$ and $\pi \pi^{*}$ states in ab initio computations}

\section{S2.1. States Identification}

The identification of the relevant states in the compounds included in the training- and test- sets has been done with the charge transfer numbers $\left(\Omega_{A B}\right)$, which quantify the hole/particle contribution of molecular fragments (A and B) in a given electronic transition, and their correlation. The lowest-lying $n \pi^{*}$ state is a localized excitation in the azo moiety. It is mainly defined as a transition from the lone-pairs of the azo nitrogen atoms to the antibonding $\pi^{*}$ system localized in the azo moiety and the surrounding rings. With our current fragment definition (see Table S1), this state is characterized by a large $\Omega_{A z o}^{n \pi^{*}}$, and smaller $\Omega_{A z O \rightarrow \mathrm{R}_{1}}^{n \pi^{*}}$ and $\Omega_{A z O \rightarrow \mathrm{R}_{2}}^{n \pi^{*}}$ contributions. This picture remains constant along our dataset. A completely different scenario is found for the target $\pi \pi^{*}$ state. This excitation is mainly a transition from the delocalized $\pi$-system to the azo, which thus holds a large part of the particle distribution (see Figure S2.1a). This is the main characteristic that differentiates the productive $\pi \pi^{*}$ state from other low-lying $\pi \pi^{*}$ states (e.g. an intruder $\pi \pi^{*}$ state localized in any of the fragments, see Figure S2.1b), and has been exploited (together with its bright nature) to identify it within the excited states manifold resulting from our quantum chemistry computations. In 5 cases (C1-6-7-7, C3-2-10-2, C3-3-9-4, C3-7-6-7 and C-3-9-0-1) computations yielded two $\pi \pi^{*}$ states that are almost degenerate, have a very similar fosc, and their character is mixed, so that both qualify as our target state (compare Figure S2.1c with S2.1d). These problematic molecules have been removed from the database, leading to a total of 3427 molecules. All raw data, including the lists of molecules included in each set (training, test and out-of-sample), have been deposited in a Materials Cloud repository.

(a)

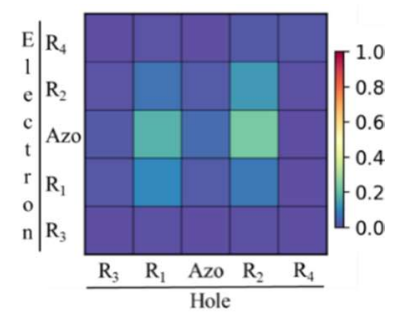

(b)

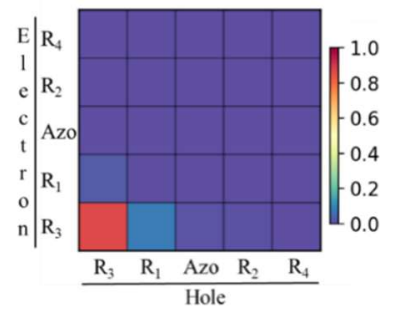

(c)

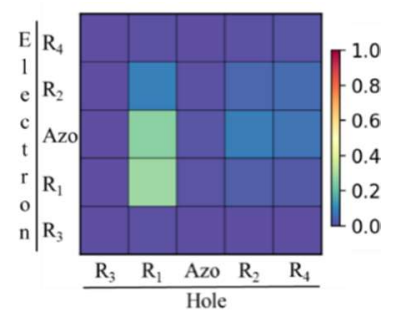

(d)

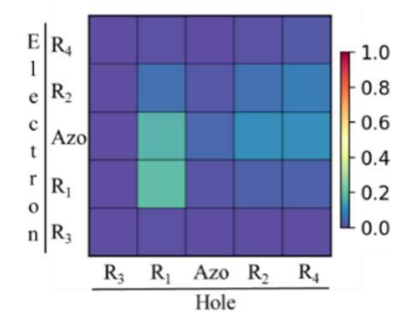

Figure S2.1. (a) Average electronic fingerprint (EF) associated with the $\pi \pi^{*}$ state within the training set. (b) An example of a non-productive, low-lying $\pi \pi^{*}$ state mainly localized in an outer fragment. Example of EF for a problematic molecule: (c) $\mathrm{S}_{2}$ and (d) $\mathrm{S}_{3}$ of compound C3-3-9-4, with energies 3.9 and $4.1 \mathrm{eV}$, both with $f_{0 s c}=0.5$.

\section{S2.2. Descriptor Space}

$A b$ initio results on the train + test sets confirm that the descriptor space is well covered by the compounds included in the training and test sets. The $\pi \pi^{*}$ transition ranges from an almost-symmetric contribution localized in both $R_{1}$ and $R_{2}$ to a similar extent, to a completely asymmetric dominant contribution of either the left $\left(\mathrm{R}_{1}+\mathrm{R}_{3}\right)$ or right $\left(\mathrm{R}_{2}+\mathrm{R}_{4}\right)$ sides of the molecule. The latter point can be seen in the distribution of the Hole and Particle densities associated with the $\pi \pi^{*}$ transition in Figure S2.2. The Hole (blue points) is distributed from $80 \%$ to $5 \%$ in the right side, and from $90 \%$ to $8 \%$ in the left side. Notice that the right and left contributions do not sum 1 because there is a small contribution from the azo fragment itself. The particle localization changes as well (red points), but the azo contribution is here more important and, hence, the variation is limited from $20 \%$ to $70 \%$.

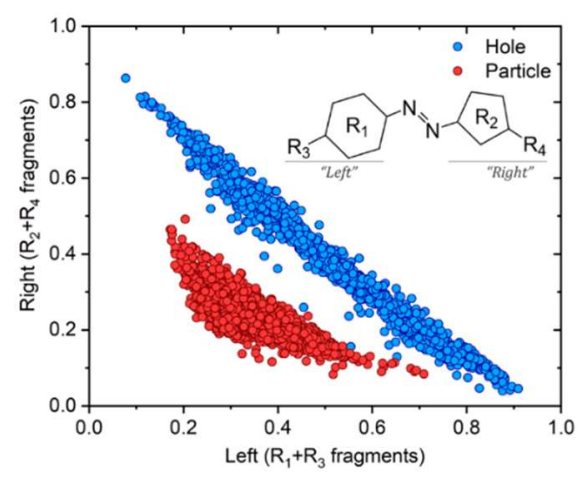

Figure S2.2. Hole and particle distributions in the left- and right- sides (see inset) of the molecules included in the train + test sets (1300 +200 molecules), associated with the productive $\pi \pi^{*}$ state. 


\section{S3. Details on the Machine Learning Framework}

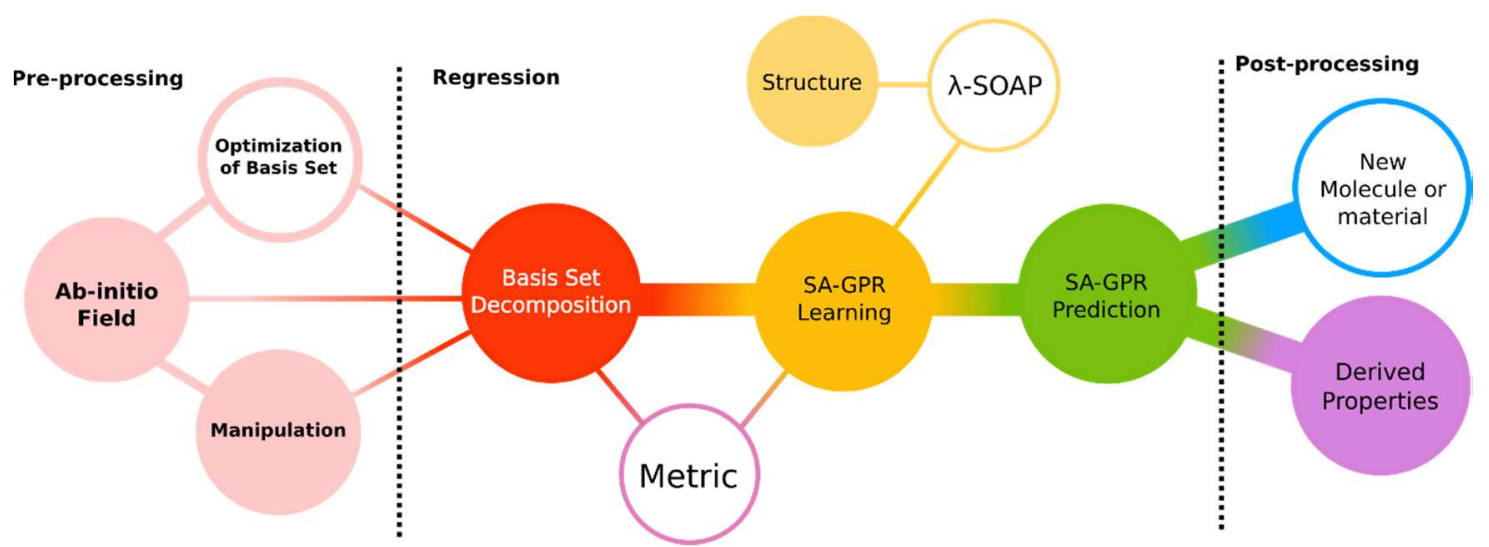

Scheme S3. General workflow connecting the identification of a field of interest in quantum chemistry with is manipulation, training, and analysis. Most aspects are discussed in this manuscript (filled circles), while the rest (open circles) can be found in the literature.

The learning of the Hole and Particle densities described in this manuscript exploits a general workflow that aims at predicting fields of interest in quantum chemistry. The workflow is divided in three main steps: (i) pre-processing, (ii) regression and post-processing (see Scheme S3). It starts with the identification of the field, which in this case is the transition density (TD). As we explain below, the TD is defined up to a sign, and thus is a poor object for machinelearning purposes. Thus, the field must be manipulated to generate equivalent objects that can be learned, in this case the separate Hole and Particle densities. This manipulation is described below, in sections S3.1 and S3.2. Details on the metric and the molecular representation can be found in reference 4 of the ESI, and in references 24-26 of the main text, respectively. The SA-GPR learning is discussed in Sections S3.3 and S4. The prediction is discussed in sections S5 and S6, together with the derived topological properties. The more relevant aspects of each step are discussed in the main text.

\section{S3.1. Learning the Hole and Particle Densities, $\rho_{H / P}(\boldsymbol{r})$.}

All the information about an excited state $l$ is contained in the transition density matrix $(X)$. The Tamm-Dancoff approximation (TDA) leads to a particularly simple form of the Casida's equations that does not depend on the virtualoccupied orbital rotations (commonly identified with the symbol $\mathbf{Y}$ ), but only on the occupied-virtual ones $(\mathbf{X}):^{1,2}$

$$
\boldsymbol{A} X_{l}=\omega_{T D A, l} X_{l}
$$

where $\mathbf{A}$ is a Hessian matrix often referred to as the excitation matrix and $\omega_{T D A, l}$ are the vertical excitation energies within the approximation. The application of TDA does not change the character nor the observables of an excited state and offers a framework where all the information is encoded in a single quantum chemical object, the matrix $\mathbf{X}$.

While the information contained in $X_{l}$ is highly valuable, the transition density matrix is not compatible with our learning framework as its real-space representation is 6-dimensional (independent hole and particle coordinates) and its sign is not uniquely defined. ${ }^{3}$ It is thus necessary to pre-process $X_{l}$ to be able to access its information via machine learning. For simplicity, from now on we drop the subindex $l$, and what follows refers to a specific excited state.

The first set of operations consists in disentangling the hole and the particle fields from the transition density matrix, which means, in practice, to separate the occupied MO manifold (occ) from the unoccupied one (virt). By definition, the Hole $(\mathrm{H})$ and Particle $(\mathrm{P})$ density matrices are the part of the transition density matrix occurring in the occupied and virtual MOs, respectively, and are defined as: 


$$
\begin{aligned}
& X_{o c c \times o c c}^{H}=X_{o c c \times v i r t} \cdot X_{v i r t \times o c c}^{\dagger} \\
& X_{v i r t \times v i r t}^{P}=X_{v i r t \times o c c}^{\dagger} \cdot X_{o c c \times v i r t}
\end{aligned}
$$

Our framework requires the fields to be expressed in terms of a nonorthogonal, atom-centered basis. However, for computational convenience, the transition density matrix and thus the hole and particle matrices are usually evaluated by quantum chemical programs in the MO basis. For this reason a second set of pre-processing operations is needed, which consists of a MO-to-AO basis set transformation.

$$
\begin{aligned}
T_{p q}^{P-A O} & =C_{\text {virt }} X_{\text {virt } \times \text { virt }}^{P} C_{\text {virt }}^{\dagger} \\
T_{p q}^{H-A O} & =C_{o c c} X_{o c c \times o c c}^{H} C_{o c c}^{\dagger}
\end{aligned}
$$

where $C_{o c c}$ and $C_{\text {virt }}$ are the occupied and virtual MO-coefficients stemming from the DFT computation. The transformed $T_{l}^{P-A}$ and $T_{l}^{H-A O}$ are now fully compatible with our ML framework, and the target densities can be simply expressed as:

$$
\rho_{H / P}(\boldsymbol{r})=\sum_{p q}^{\mathrm{N}(\mathrm{AO})} T_{p q}^{H / P-A O} \chi_{p}(\boldsymbol{r}) \chi_{q}(\boldsymbol{r}) \approx \sum_{i}^{\mathrm{N}(\mathrm{AUX})} c_{i}^{H / P} \phi_{i}(\boldsymbol{r})
$$

where $\chi_{p / q}(\boldsymbol{r})$ are atomic orbitals, $\left\{\phi_{i}(\boldsymbol{r})\right\}$ is a density fitting auxiliary basis set and $\left\{c_{i}^{H / P}\right\}$ its expansion coefficients.

Finally, the predicted densities (for Hole, Particle, ground state and TD) are corrected a posteriori to enforce their exact integration rules $\left[e . g\right.$. to 1 for $\left.\rho_{H / P}(\boldsymbol{r})\right]$. The procedure consists in finding the set of coefficients that are the closest to the prediction, but also integrates to the correct particle number. For further details on the a posteriori correction see Ref. ${ }^{4}$ of the ESI.

Because it leads to a particularly simple form of Casida's equations (see Eq. X), and it does not change the character nor the observables of an excited state, we believe that using TDA is the most efficient and simpler choice to machine learn the excited state properties of molecules.

Nonetheless, our framework is general and could be applied to standard LR-TDDFT without any further approximations. In this case, it would be necessary to rewrite Casida's equations as:

$$
C Z=\Omega^{2} Z
$$

where $\mathbf{C}=(\mathbf{A}-\mathbf{B})^{0.5}(\mathbf{A}+\mathbf{B})(\mathbf{A}-\mathbf{B})^{0.5}, \mathbf{Z}=(\mathbf{A}-\mathbf{B})^{0.5}(\mathbf{X}+\mathbf{Y})$ and $\mathbf{B}$ is a Hessian matrix commonly referred to as the deexcitation matrix. By suitable post-processing, $\mathbf{Z}$ could be used in combination with the same model described in this work to learn the excited state properties of molecules.

\section{S3.2. Learning the Transition Density, $\rho_{T D}(\boldsymbol{r})$}

As mentioned in section S3.1 and in the main text, the transition density matrix is a poor quantum chemical property to represent and learn in real-space. Its diagonal part, the transition density $\rho_{T D}(\boldsymbol{r})$ is a much easier object to visualize, as it depends only on the three spatial coordinates, but is still plagued by an arbitrary sign problem, ${ }^{3}$ which hinders machine learning applications. To circumvent this limitation, and access $\rho_{T D}(\boldsymbol{r})$ for the $\pi \pi^{*}$ state, we applied a signfixing procedure inspired by the one proposed by Marquetand and coworkers. ${ }^{3}$ More precisely, the procedure relies on the fact that the $\pi \pi^{*}$ state particle is always localized on the azo-group, while the hole is generally elsewhere. This allows to take $\rho_{T D}(\boldsymbol{r})$ on the azo group as reference and to set a sign convention. Here, the sign of transition density 
on an atom was defined as the contribution of the auxiliary basis functions centered on this atom to the total integral of $\rho_{T D}(\boldsymbol{r})$ :

$$
\sum_{i \in N_{l e f t}} c_{i} \int \phi_{i}(\boldsymbol{r}) d^{3} \boldsymbol{r}
$$

The signs on the "left" Nitrogen atom $\left(N_{\text {left }}=\mathrm{N}\right.$ bonded to $\left.\mathrm{R}_{1}\right)$ and on the "right" Nitrogen atom (bonded to $\left.\mathrm{R}_{2}\right)$ are often opposite. To establish the reference, the sign of $\rho_{T D}(\boldsymbol{r})$ on $N_{\text {left }}$ is set to be positive. For those molecules having a negative $\rho_{T D}(\boldsymbol{r})$ on the azo group, the total field is multiplied by -1 , so that the change is reversed at all points of real-space. Such a scheme worked for the $\pi \pi^{*}$ state, but not for the $n \pi *$ state because its particle and hole both display similar fragment-based distributions with a dominant contribution of the azo-group (see Figure S2.2). As a result, the $\rho_{T D}(\boldsymbol{r})$ amplitude in this fragment is nearly zero, which makes it impossible to establish a reliable sign convention.

\section{S3.3. Coulomb Metric}

The machine learning framework that allows the prediction of the hole and particle densities, as well as of any realspace field in general, is based on two distinct loss functions. ${ }^{5}$ The first regulates the decomposition of the ab initio field into atom-centered contributions, while the second dictates the accuracy of the learning exercise. While their role within the framework differs, both loss functions share a common structure (Eq. S3.1):

$$
\text { Loss }=\int d r \int d r^{\prime} \Delta \rho(\boldsymbol{r}) \hat{O}\left(\boldsymbol{r}, \boldsymbol{r}^{\prime}\right) \Delta \rho\left(\boldsymbol{r}^{\prime}\right) \quad \text { Eq. S3.1 }
$$

where $\Delta \rho$ represent the residues between the decomposed/predicted densities and the reference $\left[\right.$ i.e. $\rho^{M L}(\boldsymbol{r})-$ $\left.\rho^{r e f}(\boldsymbol{r})\right]$, and $\hat{O}\left(\boldsymbol{r}, \boldsymbol{r}^{\prime}\right)$ is any two-electron operator. For instance, one possibility is to set $\hat{O}\left(\boldsymbol{r}, \boldsymbol{r}^{\prime}\right)=\delta\left(\boldsymbol{r}-\boldsymbol{r}^{\prime}\right)$, which can be physically interpreted as Equation S3.1 expressing the overlap between the decomposed/predicted densities and the reference. This choice of operator has been found to yield only a poor approximation to the densities and is generally rejected in favor of the Coulomb operator, that is $\hat{O}\left(\boldsymbol{r}, \boldsymbol{r}^{\prime}\right)=\left|r-r^{\prime}\right|^{-1}{ }^{6-11}$ All the results presented in this work have been generated using the Coulomb metric both for the decomposition and the learning loss function. Further details about the impact of the metric in the learning process can be found in Ref. ${ }^{4}$ of the ESI.

In the main text, we report the learning curves for all the relevant fields targeted in this work using the absolute error as a metric for the accuracy of the learning (see Figure 1). This metric is particularly appealing for the hole and particle densities, as it is independent from the baseline of the model (i.e. from the spherical average of the densities on each atom), and these fields contain the same number of particles (one) for all the molecules in the dataset.

For the sake of completeness, it is relevant to show the learning curves using a relative metric, that is:

$$
\text { Relative err. [\%] }=\frac{\text { Abs. err. }}{\left(\rho^{r e f}\left(\boldsymbol{r}_{\mathbf{1}}\right)\left|\frac{1}{r_{12}}\right| \rho^{r e f}\left(\boldsymbol{r}_{2}\right)\right)} \quad \text { Eq. S3.2 }
$$

The first thing to notice is how omitting the spherical average of the atomic densities (baseline) from the computation of relative metric reflects very well the difficulty of the learning exercise. In particular, the left panel of Figure S3.2 shows that the hardest fields to learn are the hole and particle of the $\pi \pi^{*}$ state, while the easiest is the ground state density. Adding back the spherical averages of the atomic densities (Figure S3.1 right panel) has little impact for the hole and particle densities, while it decreases dramatically the error in ground-state. This is not surprising however, because the spherical averages of the atomic densities constitute a large part of the ground-state densities, as shown also by the success of the applications of the pro-molecular approach to the electron density. In contrast, the hole and particle densities contain only one particle each, and the atomic spherical averages barely contribute to the total field. 
At large training, the relative metric is in line with the absolute metric shown in the main text, which further demonstrates the overall robustness of the results shown.
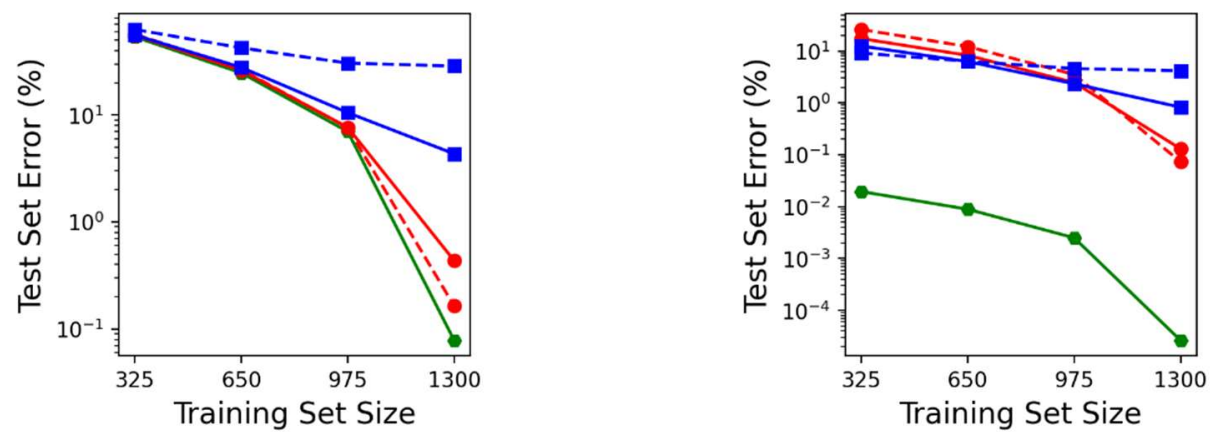

Figure S3.1. Learning Curves associated with the ground state density (green), and the Hole (dashed) and Particle (solid) of the productive $\mathrm{n} \pi^{*}$ (red) and $\pi \pi^{*}$ (blue) states. (left) Relative metric of the fields without the spherical average atomic contribution (without baseline). (right) Relative metric of the full field (baseline included). 


\section{S3.4. Visual Representation}

While absolute and relative metrics do well in objectively quantifying the error on the predicted fields, they do not allow to get an intuitive understanding of how these errors impact the shape of the densities. For this reason, we report in Figure S3.2 an illustrative example of the quality of the particle for both the $\pi \pi^{*}$ state. This molecule (C1-13-2-3) was chosen as it is characterized by an error close to the mean error of the whole test set.

Geometry

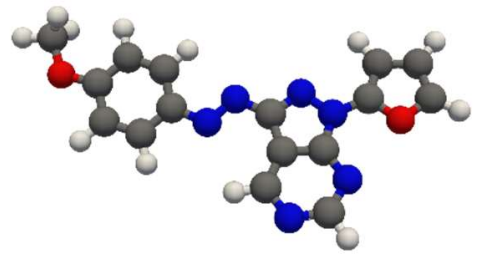

Training set Size:

325

650

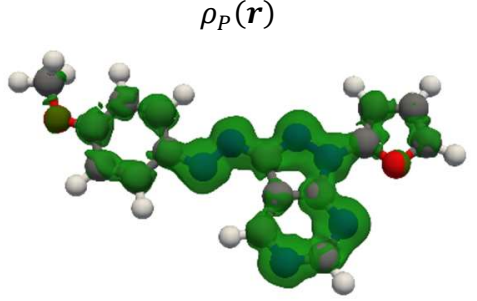

975

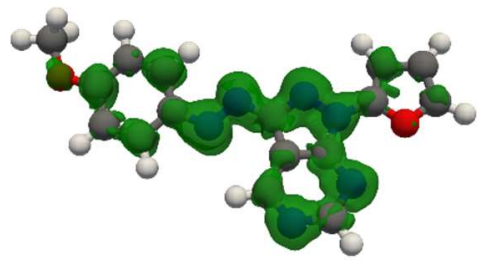

1300

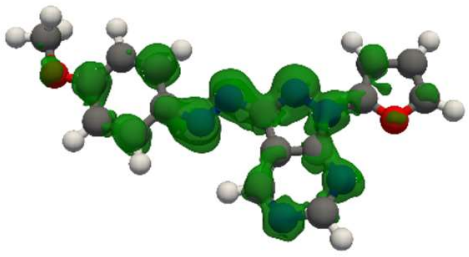

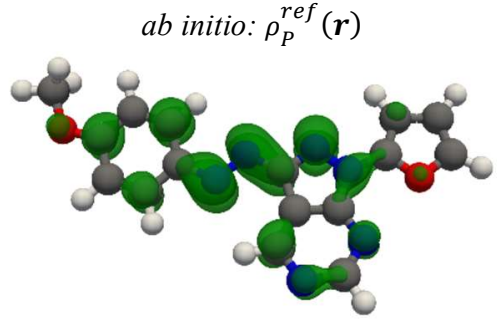
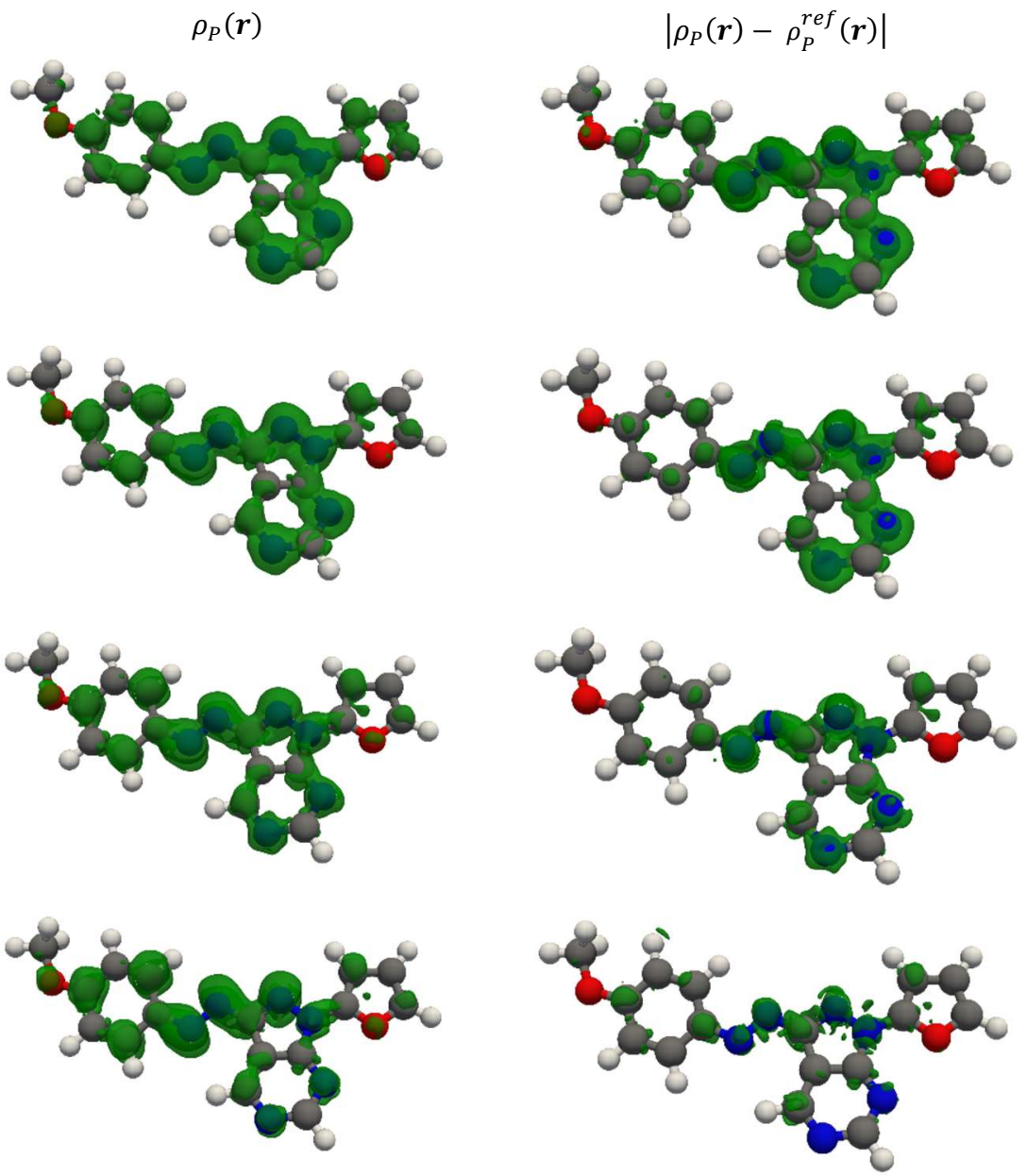

Figure S3.2. Particle distribution of the $\pi \pi^{*}$ state for compound C1-13-2-3 (isovalue $=0.001$ ). the molecular geometry, the reference (ab initio) particle density $\rho_{P}^{r e f}(\boldsymbol{r})$, and the (left) predicted density and (right) absolute density difference for at each point of the learning curve: $325,650,975$ and 1300, with an associated relative error of $10 \%, 5 \%, 2 \%$ and $1 \%$, respectively (with baseline). 

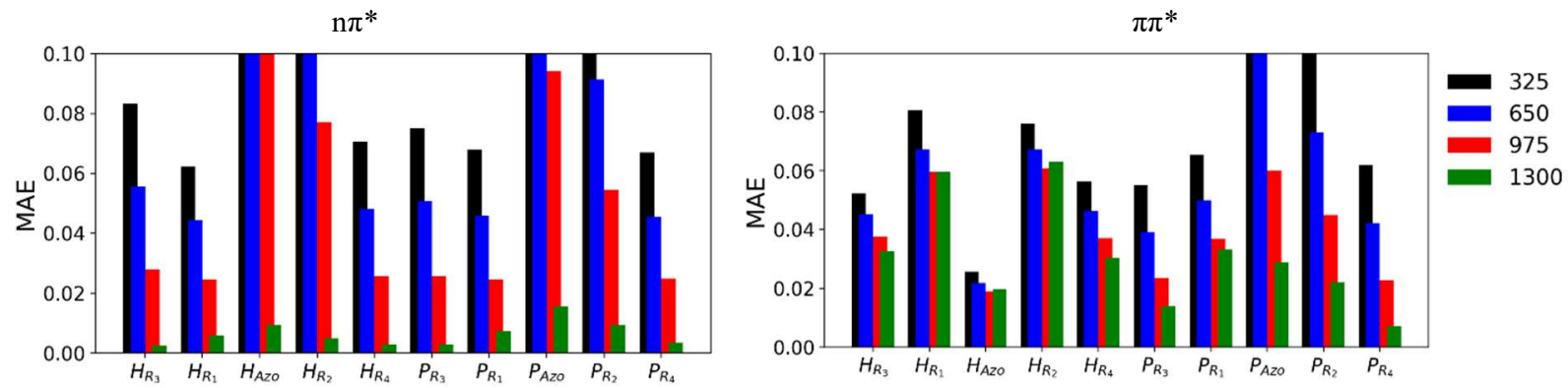

Figure S3.3. Learning Curves associated with the hole $(\mathrm{H})$ and particle $(\mathrm{P})$ densities distributed in the 5 molecular fragments (see Scheme 1) for the (left) $n \pi^{*}$ and (right) $\pi \pi^{*}$ states of the 200 molecules included in the test-set. The training set size is indicated with the color code (see legend).

The ab initio Hole (H) and Particle (P) in a given fragment are defined as: $H_{A}=\sum_{B} \Omega_{A \rightarrow B}$ and $P_{A}=\sum_{B} \Omega_{B \rightarrow A}$ and, thus, depend on the $\Omega_{A \rightarrow B}^{l}$ values extracted from an ab initio computations with TheoDORE analysis tool. ${ }^{12}$ In this program, the fragment contributions $\left(\Omega_{A \rightarrow B}^{l}\right)$ are evaluated from the TDM, which in this case is expressed in the basis of atomic orbitals. In contrast, the assignment of predicted densities in real-space to specific fragments is based on the dominant Hirschfeld partitioning. It thus follows that $a b$ initio and predicted values in our plots result from a different way of extracting the fragment-based contributions $\left(X_{R_{1}-R_{4}}\right)$. To ensure that this does not contribute to the error, we plot below the correlation between the ab initio values extracted from TheoDORE and summed into $H_{A}$ and $P_{A}$ (see above), with those resulting from a Hirschfeld partitioning of the ab initio density. In general, the error is minor $(0.4 \%$ in average), and it reaches $0.7 \%$ in the worst cases, such as the $P_{A z o}^{n \pi^{*}}$ and $P_{A z o}^{\pi \pi^{*}}$ contributions shown in Figure S3.4.
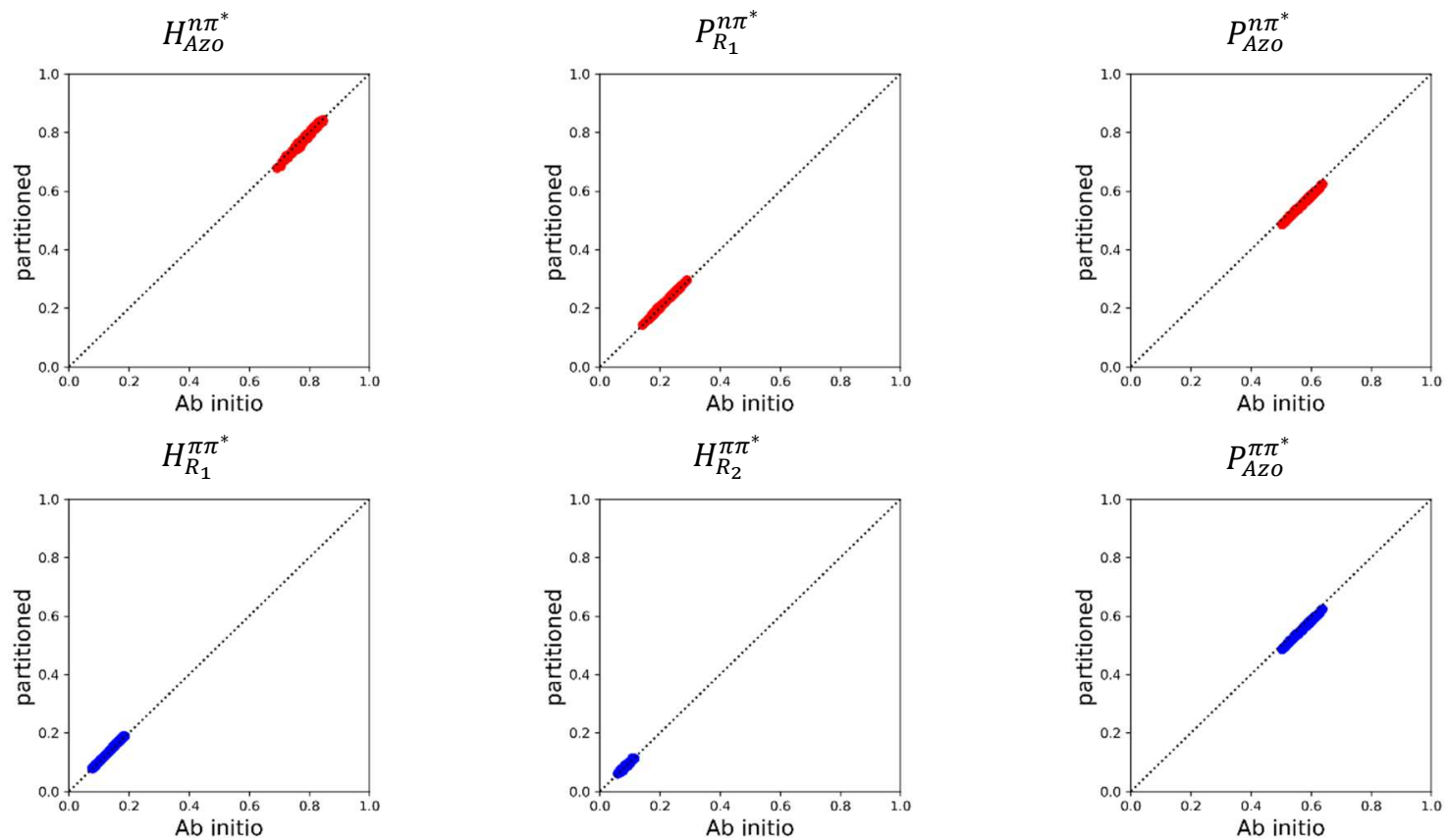

Figure S3.4. Comparison between the $a b$ initio values of some relevant densities of the $n \pi^{*}$ (top, red) and $\pi \pi^{*}$ (below, blue) bands, extracted from either the $\Omega_{A B}$ values with TheoDORE (ab initio), or from the partitioning of the real-space H/P density (partitioned), for the 200 molecules included in the test set. 


\title{
S4. Comparison of Predicted vs. ab initio Descriptors
}

\author{
S4.1. Hole and Particle Distributions
}

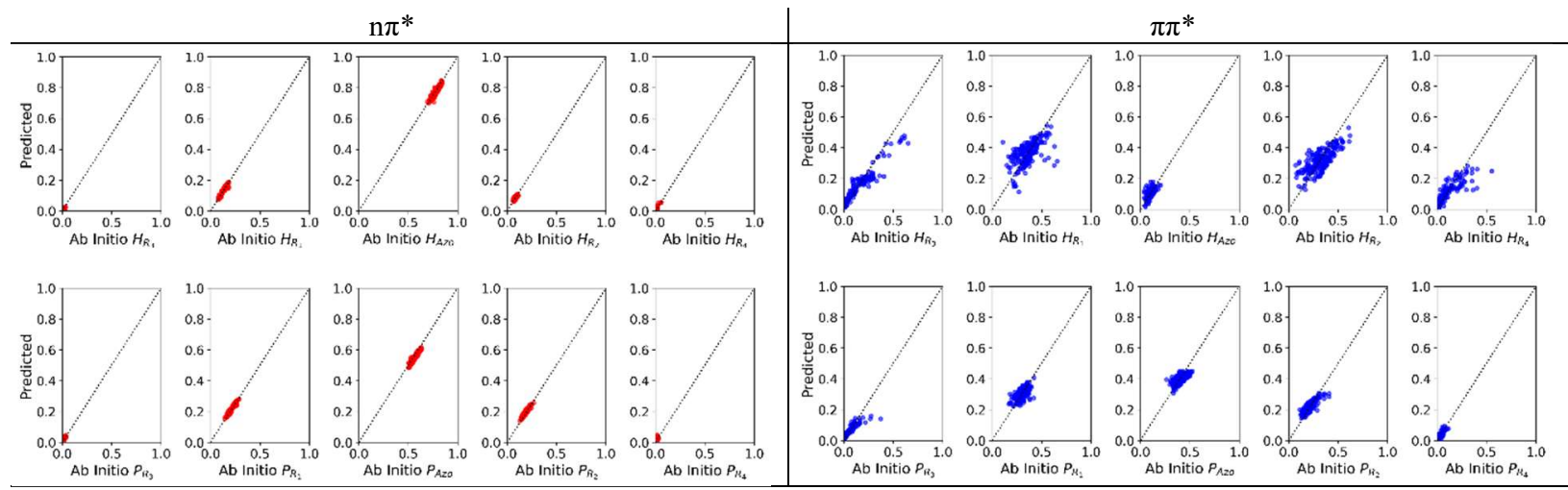

Figure S4.1. Comparison between predicted and ab initio hole (H, top) and particle (P, below) densities distributed in the 5 molecular fragments for the (left) $n \pi^{*}$ and (right) $\pi \pi^{*}$ states of the 200 molecules included in the test-set. The results correspond to the ML model with 1300 molecules in the training set.

S4.2. Non-local nature of the $\pi \pi^{*}$ state, and impact on the predicted $H_{R_{1}}^{\pi \pi^{*}}$ values

a) All
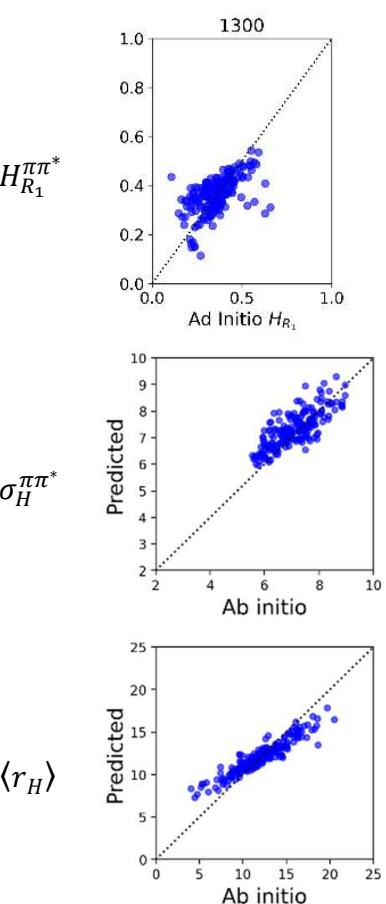

b) $\mathrm{R}_{3}$ and $\mathrm{R}_{4}$ non $\pi$-conjugated
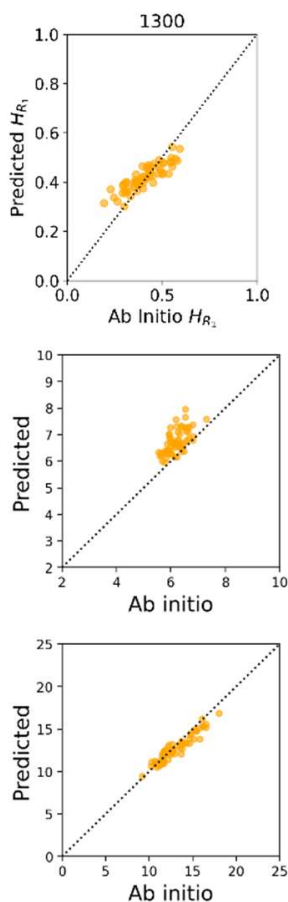

c) $R_{3}$ is $\pi$ $\left(H_{R_{1}}^{\pi \pi^{*}}\right.$ is local $)$
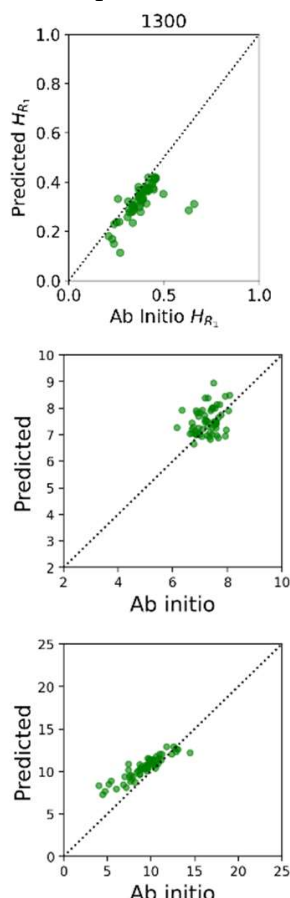

d) $R_{4}$ is $\pi$ $\left(H_{R_{1}}^{\pi \pi^{*}}\right.$ is non-local)
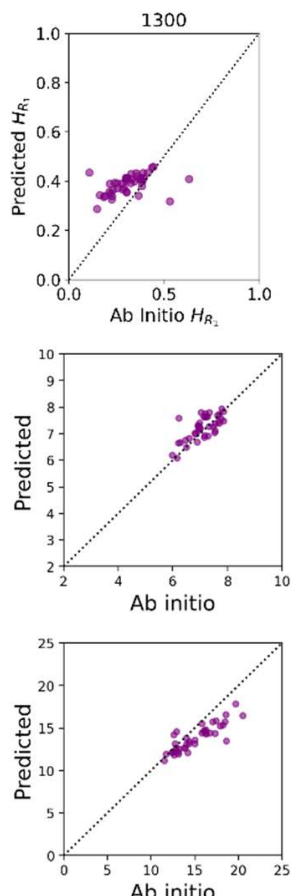

e) Both are $\pi$
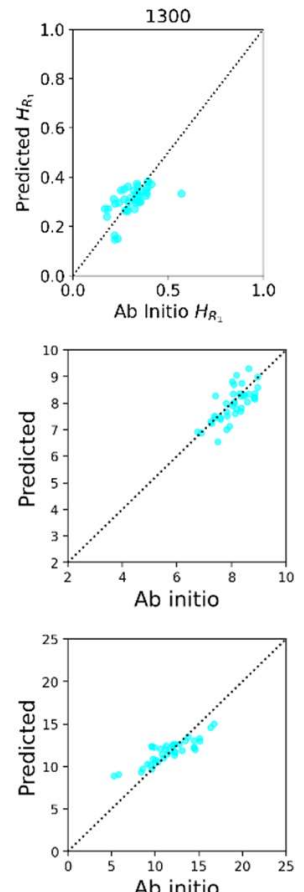

Figure S4.2. Comparison of ab initio and predicted (top) $H_{R_{1}}^{\pi \pi^{*}}$, (middle) $\sigma_{H}^{\pi \pi^{*}}$, and (bottom) $\left\langle r_{H}\right\rangle$ for the 200 molecules included in the test set. Five sets are shown: (a) All compounds. (b) Those with non- $\pi$-conjugated fragments as $\mathrm{R}_{3}$ and $\mathrm{R}_{4}$. (c) Those with $\pi$-conjugated fragments in $\mathrm{R}_{3}$ and a non-conjugated one in $\mathrm{R}_{4}$. (d) Reverse case. (e) Those with $\pi$-conjugated fragments in both $\mathrm{R}_{3}$ and $\mathrm{R}_{4}$. Notice that small $\left\langle r_{H}\right\rangle$ values refer to the left-hand side of the molecule (fragments $\mathrm{R}_{3}+\mathrm{R}_{1}$ ), while large values refer to the right-hand side (fragments $\mathrm{R}_{2}+\mathrm{R}_{4}$ ), as oriented in Scheme 1.

The quality of the learning of $\rho_{H / P}(\boldsymbol{r})$ depends on how much is the density determined by the neighboring atoms. The case of $H_{R_{1}}^{\pi \pi^{*}}$ is an excellent example to illustrate this point (see Figure S4.2-top). 
- When both $\mathrm{R}_{3}$ and $\mathrm{R}_{4}$ are non $\pi$-conjugated fragments (see Figure $\mathrm{S} 4.2 \mathrm{~b}$ ), $\rho_{H}(\boldsymbol{r})$ is mostly concentrated in the $\mathrm{R}_{1}$ Azo- $R_{2}$ region. The hole distribution is thus mostly determined by the competition between $R_{1}$ and the non-adjacent $\mathrm{R}_{2}$. In these circumstances, the ML model struggles to assign the $\mathrm{R}_{1}$ contribution because is not defined by the local environment, and opts to delocalize the density in both fragments, resulting in a flatter slope. Notice that $\left\langle r_{H}\right\rangle$ shows an excellent correlation (good position), but $\sigma_{H}^{\pi \pi^{*}}$ tends to be larger (delocalization).

- When $R_{3}$ is a $\pi$-conjugated fragment, and $R_{4}$ is not (see Figure $S 4.2 c$ ), the hole is mostly concentrated in the $R_{3}-R_{1}$ Azo region. Hence, the Hole distribution in $\mathrm{R}_{1}$ competes with the adjacent $\mathrm{R}_{3}$ fragment. In this case, the model is able to read the local environment and correctly distribute $H_{R_{1}}^{\pi \pi^{*}}$, hence the better correlation.

- The worst correlation is found when $R_{4}$ is a $\pi$-conjugated fragment, and $R_{3}$ is not (see Figure $S 4.2 d$ ). In this case, the ab initio hole concentrates in the right-hand side of the molecule $\left(\mathrm{R}_{2}+\mathrm{R}_{4}\right)$, which is associated with large $\left\langle r_{H}\right\rangle$ values (see Figure S4.2d-bottom), but the ML model once again struggles at capturing $H_{R_{1}}^{\pi \pi^{*}}$, because it is based on the chemical composition of far-away fragments, and opts to delocalize.

This illustrates the problems associated with the fact that electronic excitations are not local; the contribution of a given fragment to any of the quasiparticles (Particle or Hole) does not depend on its local environment. Finally, one must keep in mind that $\sigma_{H}^{\pi \pi^{*}}$ and $\left\langle r_{H}\right\rangle$ refer to the whole density, while $H_{R_{1}}^{\pi \pi^{*}}$ (the top row) is only one portion of it. Good $H_{R_{1}}^{\pi \pi^{*}}$ correlations are not necessarily related with a better overall Hole distribution, as we show in the $\sigma_{H}^{\pi \pi^{*}}$ panel. 


\section{S4.3. Topological Descriptors}
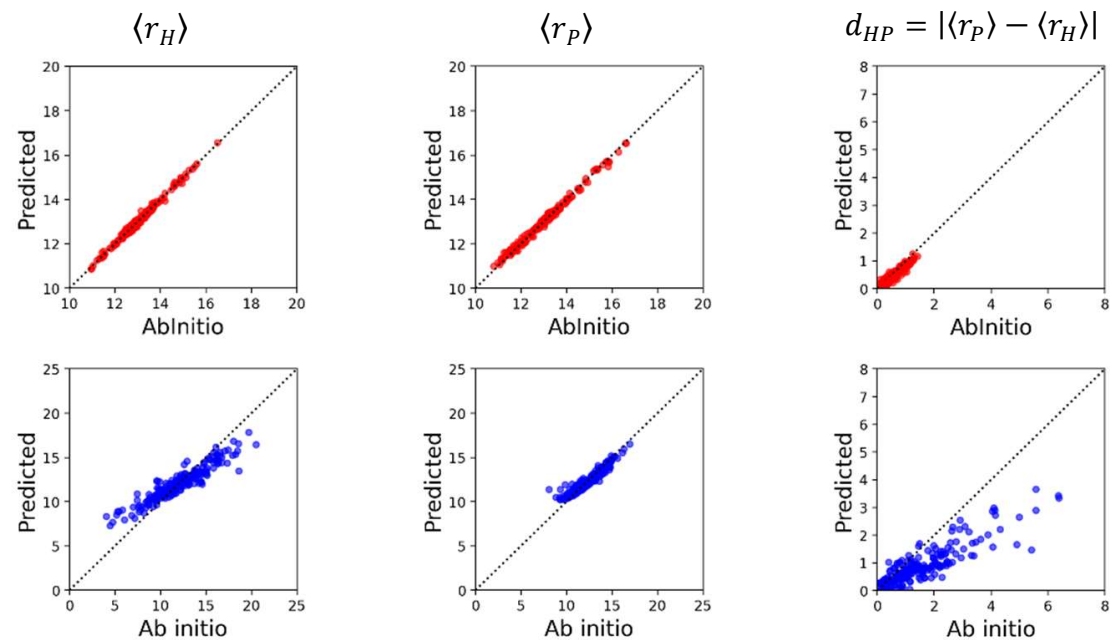

Figure S4.3. Comparison between the predicted and ab initio (left) Hole expectation value, (middle) particle expectation value, and (right) $d_{H P}$ for the (top) $n \pi^{*}$ and (below) $\pi \pi^{*}$ states.

For the $\pi \pi^{*}$ state, the tendency of ML models to delocalize the Hole (and the Particle to a lesser extent) distribution leads to the underestimation of their expectation value (see Figure S4.3). The flatter slope of $\left\langle r_{H / P}\right\rangle$ for the $\pi \pi^{*}$ band indicates a displacement of the predicted $\mathrm{H} / \mathrm{P}$ positions with respect to the ab initio values. As a result, the exciton distance is significantly reduced.

Finally, the individual components of the transition dipole moment vector are compared below (see Figure S4.4), where it is shown that the vector is not reoriented by the ML model.
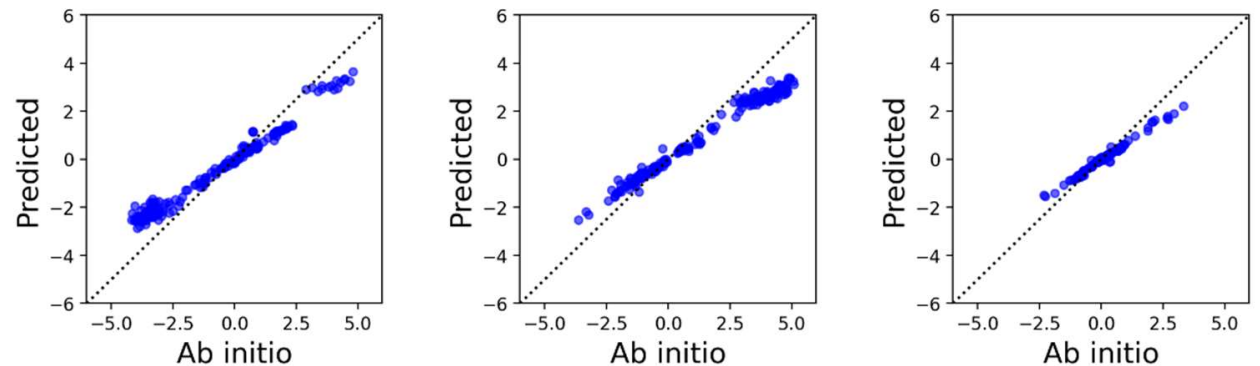

Figure S4.4. Correlation between predicted and ab initio (from left to right) $\mathrm{x}, \mathrm{y}$ and $\mathrm{z}$ components of the transition dipole of the $\pi \pi^{*}$ state 


\section{S5. Analysis of the $\pi \pi *$ State Character}

\section{S5.1. Analysis of hole and particle contributions of the $R_{3}$ fragment}

As discussed in the main text, the Hole distribution of the $\pi \pi^{*}$ state depends on (i) the extension of the $\pi$-conjugation, and (ii) the electronic properties of the fragments. On the one hand, $\pi$-type fragments in $\mathrm{R}_{3}$ maximize their Hole $\left(H_{R_{3}}^{\pi \pi^{*}}\right)$ and Particle $\left(P_{R_{3}}^{\pi \pi^{*}}\right)$ contribution, due to the mixing of their $\pi$-orbitals with those of the $\mathrm{R}_{1}-\mathrm{Azo}-\mathrm{R}_{2}$ core. As can be seen in Figure S5.1, this is the main pattern that explains both $H_{R_{3}}^{\pi \pi^{*}}$ and $P_{R_{3}}^{\pi \pi^{*}}$. Then, within the $\pi$ and non- $\pi$ conjugated subsets, $H_{R_{3}}^{\pi \pi^{*}}$ depends on the electron-withdrawing/-donating character of the fragments. For each subset, the average $H_{R_{3}}^{\pi \pi^{*}}$ contribution evolves as:

non- $\pi$ : $\mathrm{NH}_{2}>\mathrm{OMe}>\mathrm{CN}>\mathrm{Me}>\mathrm{F}>\mathrm{H}$

$\pi$ : Thiazine $>4$-im $\approx$ Furane $\approx$ Thiophene $>1-\mathrm{pz}$
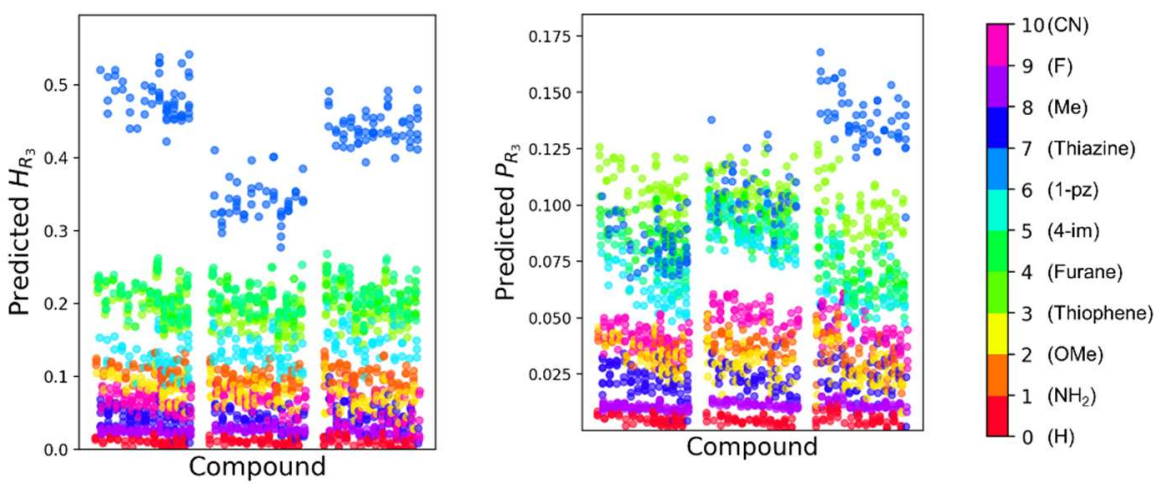

Figure S5.1. (left) Hole and (right) Particle distribution in the $\mathrm{R}_{3}$ fragment for the compounds in the out-of-sample set (1927 molecules). Each plot shows the compounds organized in the Xaxis, with the 3 blocks of data being $R_{1}=1-3$, while the color code (see color bar) represents the $\mathrm{R}_{3}$ fragment. 


\section{S5.2. Tetrazole- and Adenine-based compounds}

As discussed in the main text and in the section above, the Hole distribution of the $\pi \pi^{*}$ state depends on the push-pull character of the fragments. The impact of $\mathrm{R}_{1}$ has been discussed above, but other fragments are also able to modulate it. In figure $\mathrm{S} 5.2$, we observe the impact of the $\mathrm{R}_{2}$ fragment on its associated Hole $\left(H_{R_{2}}^{\pi \pi^{*}}\right)$ and Particle $\left(P_{R_{2}}^{\pi \pi^{*}}\right)$. Tetrazole $\left(\mathrm{R}_{2}=9\right)$ displays much smaller $H_{R_{2}}^{\pi \pi^{*}}$ and $P_{R_{2}}^{\pi \pi^{*}}$ than other fragments, due to its poor donor- and acceptor capabilities. Similarly, adenine $\left(\mathrm{R}_{2}=13\right)$ displays the largest $P_{R_{2}}^{\pi \pi^{*}}$, in agreement with its good acceptor character, and a moderate $H_{R_{2}}^{\pi \pi^{*}}$. The donor and acceptor capabilities of the fragments are quantified in Section S5.3.
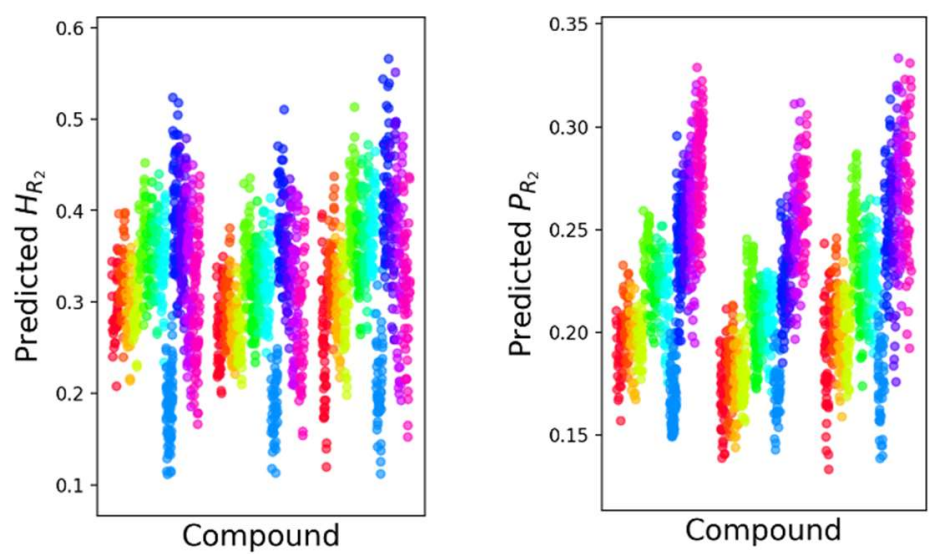

$\mathrm{R}_{2}$

Figure S5.2. Predicted (left) Hole and (right) Particle distribution of the $\pi \pi^{*}$ state in the $\mathrm{R}_{2}$ fragment $\left(H_{R_{2}}^{\pi \pi^{*}}\right.$ and $\left.P_{R_{2}}^{\pi \pi^{*}}\right)$ for the compounds in the out-of-sample set (1927 molecules). Each plot shows the compounds organized in the $\mathrm{X}$-axis, with the 3 blocks of data being $\mathrm{R}_{1}=1-3$, while the color code represents the $\mathrm{R}_{2}$ fragment. 


\section{S6. Analysis of the $n \pi *$ State Character}

\section{S6.1. Impact of the $R_{1}$ fragment on the State Character}

The main pattern governing the $n \pi^{*}$ state character is the amount of $\mathrm{H} / \mathrm{P}$ in the Azo group $v s$. the neighboring fragments $\left(\mathrm{R}_{1}\right.$ and $\left.\mathrm{R}_{2}\right)$, ${ }^{13}$ whose balance is dictated by the fragments electronic properties. In the case of $\mathrm{R}_{1}$, systems with Thiadiazole ( $\mathrm{R}_{1}=3$ fragment) are always associated with a larger Hole contribution than when Benzene $\left(\mathrm{R}_{1}=1\right)$ or Thiazole $\left(\mathrm{R}_{1}=2\right)$ are used instead (see Figure S6.1). This tendency correlates with the capacity of these fragments as donors, as quantified by the ionization potential (IP), which is $8.9,8.6$ and $9.7 \mathrm{eV}$ for $\mathrm{R}_{1-3}$, respectively.

(a) $H_{R_{1}}^{n \pi^{*}}$

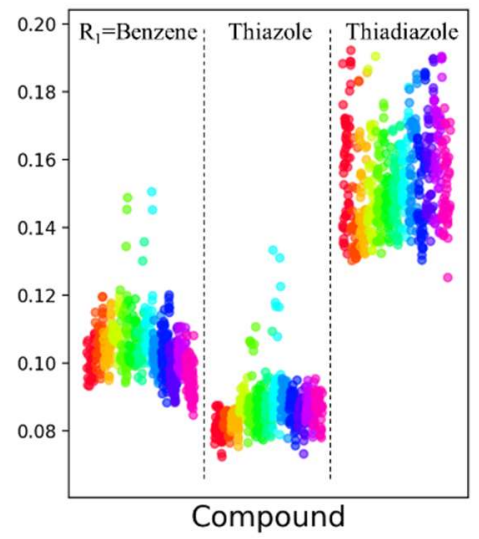

$\mathrm{R}_{2}$

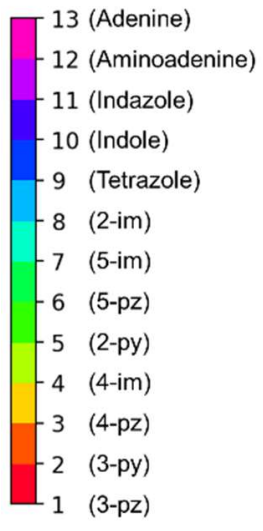

Figure S6.1. Predicted Hole in (a) fragment $R_{1}$ and (b) in the azo group, for the compounds in the out-ofsample set (1927 dyes). Each plot shows the compounds organized in the X-axis, with the 3 blocks of data being $\mathrm{R}_{1}=1-3$ (Benzene, Thiazole, Thiadiazole, see inset), while the color code represents the $\mathrm{R}_{2}$ fragment.

\section{References}

1. Casida, M. E.; Gutierrez, F.; Guan, J.; Gadea, F.-X.; Salahub, D.; Daudey, J.-P. Charge-Transfer Correction for Improved TimeDependent Local Density Approximation Excited-State Potential Energy Curves: Analysis within the Two-Level Model with Illustration for $\mathrm{H} 2$ and Lih. J. Chem. Phys. 2000, 113, 7062-7071.

2. Hirata, S.; Head-Gordon, M. Time-Dependent Density Functional Theory within the Tamm-Dancoff Approximation. Chem. Phys. Lett. 1999, 314, 291-299.

3. Westermayr, J.; Gastegger, M.; Menger, M. F. S. J.; Mai, S.; González, L.; Marquetand, P. Machine Learning Enables Long Time Scale Molecular Photodynamics Simulations. Chem. Sci. 2019, 10, 8100-8107.

4. Briling, K. R.; Fabrizio, A.; Corminboeuf, C. Impact of Quantum-Chemical Metrics on the Machine Learning Prediction of Electron Density. arXiv 2021, 2104.12457.

5. Fabrizio, A.; Grisafi, A.; Meyer, B.; Ceriotti, M.; Corminboeuf, C. Electron Density Learning of Non-Covalent Systems. Chem. Sci. 2019, 10, 9424-9432.

6. Whitten, J. L. Coulombic Potential Energy Integrals and Approximations. J. Chem. Phys. 1973, 58, 4496-4501.

7. Dunlap, B. I.; Connolly, J. W. D.; Sabin, J. R. On First-Row Diatomic Molecules and Local Density Models. J. Chem. Phys. 1979, 71, 4993-4999.

8. Dunlap, B. I.; Connolly, J. W. D.; Sabin, J. R. On Some Approximations in Applications of Xa Theory. J. Chem. Phys. 1979, 71,3396-3402.

9. Hall, G. G.; Smith, C. M. Fitting Electron Densities of Molecules. Int. J. Quantum Chem. 1984, 25, 881-890.

10.Smith, C. M.; Hall, G. G. The Approximation of Electron Densities. Theor. Chim. Acta 1986, 69, 63-69.

11.Eichkorn, K.; Treutler, O.; Öhm, H.; Häser, M.; Ahlrichs, R. Auxiliary Basis Sets to Approximate Coulomb Potentials. Chem. Phys. Lett. 1995, 240, 283-290.

12.Plasser, F. Theodore: A Toolbox for a Detailed and Automated Analysis of Electronic Excited State Computations. J. Chem. Phys. 2020, 152, 084108 .

13.Vela, S.; Krüger, C.; Corminboeuf, C. Exploring Chemical Space in the Search for Improved Azoheteroarene-Based Photoswitches. Phys. Chem. Chem. Phys. 2019, 21, 20782-20790. 